PROCEEDINGS OF THE

AMERICAN MATHEMATICAL SOCIETY

Volume 138, Number 9, September 2010, Pages 3323-3334

S 0002-9939(2010)10399-4

Article electronically published on May 17, 2010

\title{
SATURATION OF THE WEAKLY COMPACT IDEAL
}

\author{
ALEX HELLSTEN
}

(Communicated by Julia Knight)

\begin{abstract}
It is consistent relative to a measurable cardinal of order $\alpha$ that the ideal associated with a weakly compact cardinal of order $\alpha$ is saturated.
\end{abstract}

\section{INTRODUCTION}

In 5 greatly $\Pi_{1}^{1}$-indescribable cardinals were introduced and it was shown that the weakly compact ideal on such a cardinal is not saturated. This is analogous to a result of Baumgartner, Taylor, and Wagon [2] stating that $\mathrm{NS}_{\kappa} \mid \operatorname{Reg}$ is not $\kappa^{+}$saturated when $\kappa$ is greatly Mahlo. An equiconsistency result is proved in Jech and Woodin [6] by means of a forcing construction. Starting with a ground model with an $\alpha$-measurable cardinal $\kappa$, one obtains a generic extension where $\kappa$ is $\alpha$-Mahlo and $\mathrm{NS}_{\kappa} \mid$ Reg is $\kappa^{+}$-saturated. This can be done for any ordinal $\alpha<\kappa^{+}$, pointing to the fact that the above-mentioned result of $[2$ is the best possible in the sense of the Mahlo hierarchy. By Gitik and Shelah [3], restricting to the regular cardinals is necessary.

In this paper we modify the forcing construction of [6] so that we obtain a model where $\kappa$ is weakly compact of order $\alpha$ and the weakly compact ideal on $\kappa$ is $\kappa^{+}$-saturated. See [5] for a definition of the order of weakly compact, i.e. $\Pi_{1}^{1}$ indescribable, cardinals and sets. As the weakly compact ideal $\Pi_{1}^{1}(\kappa)$ is normal and $\mathrm{NS}_{\kappa} \mid \operatorname{Reg} \subseteq \Pi_{1}^{1}(\kappa)$, the first impression is that $\Pi_{1}^{1}(\kappa)$ is $\kappa^{+}$-saturated in the model of [6]. This is indeed true but for trivial reasons. In the model of [6], $\kappa$ cannot be weakly compact; i.e. we must have $\Pi_{1}^{1}(\kappa)=\mathcal{P}(\kappa)$ by results of [2].

Note that our modified forcing construction works for $\Pi_{1}^{1}(\kappa)$ without restricting the ideal, and thus the result we obtain shows that the fact that $\Pi_{1}^{1}(\kappa)$ is not $\kappa^{+}$saturated on a greatly $\Pi_{1}^{1}$-indescribable cardinal $\kappa$ is the best possible result in terms of the weakly compact hierarchy. Whereas the results of 5 readily generalise to the ideal $\Pi_{n}^{1}(\kappa)$ for $n<\omega$, it is not clear whether the result of this article generalises in the same way even to $\Pi_{2}^{1}(\kappa)$.

The bulk of this paper consists of a detailed proof of the following result, where the order $\alpha$ of the measurable and the weakly compact is 1 .

Theorem 1. It is consistent relative to a measurable cardinal that there exists a weakly compact cardinal $\kappa$ on which the weakly compact ideal is $\kappa^{+}$-saturated.

Our basic tool is the forcing notion from [4, shooting a 1-club through a weakly compact set. Apart from this our forcing notion is similar to that of [], although we

Received by the editors October 27, 2006 and, in revised form, January 21, 2010.

2010 Mathematics Subject Classification. Primary 03E55, 03 E35.

(C)2010 American Mathematical Society 3323

Reverts to public domain 28 years from publication 
have chosen a rather different way to present the technical details of the iteration. But preservation of weak compactness is the main issue when shooting 1-clubs and this requires new arguments, since similar considerations are not needed when shooting clubs. The iteration of [6] does not preserve weak compactness, as was implicitly noted above.

Definitions of concepts that are not commonly used in set-theoretical expositions are presented in Section 2 along with some other preliminaries. We try to keep the paper somewhat self-contained so that the definition of the forcing notion and the technical details in the proofs can be followed without consulting the references.

We also wish to announce the following vaguely related fact. The problem mentioned in [5] is only of interest in the case of weak $\Pi_{1}^{1}$-indescribability since the following is a mere observation given Tarski's tree construction [8] and the fact that any $\Pi_{n}^{1}$-indescribable set may be split into two disjoint $\Pi_{n}^{1}$-indescribable sets.

Theorem 2 (Solovay, Tarski). For $n<\omega$ the $\Pi_{n}^{1}$-indescribable ideal over $\kappa$ is nowhere $\kappa$-saturated.

\section{WEAKLY COMPACT SETS}

As in the standard definition of a $\Pi_{1}^{1}$-indescribable cardinal, a $\Pi_{1}^{1}$-sentence is a sentence in the language of set theory with one unary predicate added. The second-order variable is also unary. A subset $E$ of a regular cardinal $\kappa$ is weakly compact if for every $\Pi_{1}^{1}$-sentence $\phi$ and every $U \subseteq V_{\kappa}$ such that $\left\langle V_{\kappa}, \in, U\right\rangle \models \phi$ there exists an ordinal $\alpha \in E$ such that $\left\langle V_{\alpha}, \in, U \cap V_{\alpha}\right\rangle \models \phi$. Such a set is also called $\Pi_{1}^{1}$-indescribable. The standard definition of a weakly compact cardinal coincides with $\kappa$ being weakly compact as a subset of itself.

The weakly compact ideal on a regular cardinal $\kappa$ is the collection of all subsets of $\kappa$ that are not weakly compact. This collection is denoted $\Pi_{1}^{1}$ or sometimes $\Pi_{1}^{1}(\kappa)$ if $\kappa$ is not clear from the context. It is a normal ideal over $\kappa$ and is also called the $\Pi_{1}^{1}$-indescribable ideal. As we admit $\mathcal{P}(\kappa)$ as a normal ideal, this holds for every regular $\kappa$, and $\kappa$ is weakly compact as a cardinal iff the weakly compact ideal is proper i.e. iff $\Pi_{1}^{1} \neq \mathcal{P}(\kappa)$.

Let $\kappa$ be an inaccessible cardinal. A subset $X$ of $\kappa$ is 1 -closed if $\alpha \in X$ whenever $\alpha<\kappa$ is inaccessible and $X \cap \alpha$ is stationary in $\alpha$. If $X$ is both 1-closed and stationary in $\kappa$, then $X$ is said to be a 1-club. By a very slight modification of a result from [7] (see [4] or [5]) a subset of a weakly compact cardinal $\kappa$ is in the weakly compact ideal if and only if its complement contains a 1-club.

The proof of the result mentioned above is rather simple and we shall sketch the main ideas. For the easier direction we note that any 1-club $X$ contains the set $\left\{\alpha \in \kappa:\left\langle V_{\alpha}, \in, X \cap \alpha\right\rangle \models \phi\right\}$, where $\phi$ expresses that the predicate $X \cap \alpha$ is stationary and $\alpha$ is inaccessible. For the other direction one uses the fact that the failure of $\left\langle V_{\alpha}, \in, U \cap V_{\alpha}\right\rangle \models \phi$ for $U \subseteq V_{\kappa}$ and a $\Pi_{1}^{1}$-sentence $\phi$ can be expressed by a first-order sentence using predicates. This is the key fact since a first-order statement reflects down to a closed unbounded subset of $\alpha$.

For an ideal $I$ over a regular cardinal $\kappa$, a subset of $\kappa$ is said to have positive measure if it is not in $I$. Thus for $\mathrm{NS}_{\kappa}$ the sets of positive measure are the stationary sets, and for $\Pi_{1}^{1}$ the weakly compact sets. An ideal $I$ over $\kappa$ is saturated if there exists no collection $\left\{X_{\alpha}: \alpha<\kappa^{+}\right\}$of cardinality $\kappa^{+}$of sets of positive measure that are pairwise almost disjoint in the sense that $X_{\alpha} \cap X_{\beta} \in I$ whenever $\alpha<\beta<\kappa^{+}$. If $I$ is normal, then the condition $X_{\alpha} \cap X_{\beta} \in I$ can be replaced by $\sup \left(X_{\alpha} \cap X_{\beta}\right)<\kappa$. 
Thus if $J$ is a normal ideal over $\kappa,[\kappa]^{<\kappa} \subseteq I \subseteq J$, and $I$ is saturated, then $J$ is saturated.

For $E \subseteq \kappa$ we let $I \mid E=\{X \subseteq \kappa: X \cap E \in I\}$. If $I$ is a normal ideal, then so is $I \mid E$. Note that $I \subset I \mid E \subset \mathcal{P}(\kappa)$ iff both $E$ and its complement have positive measure with respect to $I$. E.g. since $\operatorname{Reg}=\{\alpha<\kappa: \operatorname{cf} \alpha=\alpha\}$ is in the weakly compact filter, the dual of $\Pi_{1}^{1}$, we have $\mathrm{NS}_{\kappa}\left|\operatorname{Reg} \subseteq \Pi_{1}^{1}\right| \operatorname{Reg}=\Pi_{1}^{1}$.

\section{The BASIC FORCING NOTION}

Let $E$ denote an unbounded subset of an inaccessible cardinal $\kappa$. The forcing notion $T_{1}(E)$ consists of all bounded 1-closed subsets of $E$ ordered by end extension. Thus $p \leq q$ iff $q=p \mid \sup _{\alpha \in q}(\alpha+1)$. If $E$ is weakly compact, then $T_{1}(E)$ can be used to shoot a 1-club through $E$, while preserving weak compactness, as was shown in [4].

Note that $T_{1}(E)$ is a tree that indeed has $\kappa$-branches even if $E$ is not in the weakly compact filter. One needs slightly more than the standard density argument to see that the new branch produced by a generic for $T_{1}(E)$ represents a stationary set and thus a 1-club, as this may not always be the case.

Let $P$ be a forcing notion. The game $\mathcal{G}_{\alpha}(P)$ is played by two players I and II that take turns picking a decreasing sequence of conditions in $P$. Player II wins if the game continues until a decreasing $\alpha$-sequence has been constructed. If at some earlier stage a decreasing sequence with no lower bound has been constructed, then the game ends in the victory of player I. Let us say that player I makes the first move and plays first at limits.

In the construction of [6] and many other similar constructions, clubs are shot through sets that contain all singular limit ordinals. The standard argument in these situations applies also to $T_{1}(E)$ : For a given $\alpha<\kappa$ the set $D=\left\{p \in T_{1}(E)\right.$ : $\left.\sup p \geq \alpha^{+}\right\}$is dense and $\alpha^{+}$-closed. The following lemma is then immediate.

Lemma 1. Player II has a winning strategy in $\mathcal{G}_{\alpha}\left(T_{1}(E)\right)$ for every $\alpha<\kappa$ and thus $T_{1}(E)$ does not add new bounded subsets of $\kappa$.

This is not quite enough for our purposes though. When attempting to shoot a club through a set of the wrong kind, cardinals are usually collapsed. An interesting feature of $T_{1}(E)$ is that it is relatively harmless regardless of the choice of $E$. The following lemma is not used in this paper, but we wish to better illustrate the behaviour of $T_{1}(E)$. By a generic branch we mean the union of some $T_{1}(E)$-generic set.

Lemma 2. If any generic branch added by $T_{1}(E)$ is nonstationary, then player II has a winning strategy in $\mathcal{G}_{\kappa}\left(T_{1}(E)\right)$.

Proof. Let $\dot{C}$ be a name for a club such that $\Vdash \cup \dot{G} \cap \dot{C}=\varnothing$ ". Suppose that $p_{i}$ is the move of player I at stage $i$ in the game and player II is to pick $q_{i} \leq p_{i}$. Together with $q_{i}$, player II picks a closed bounded subset $C_{i}$ of $\kappa$. By Lemma 1 it is possible to make sure that

(i) $\sup q_{i} \geq \max C_{i}>\sup p_{i}$

(ii) $\quad q_{i} \Vdash$ " $\check{C}_{i}$ is an initial segment of $\dot{C}$ ".

If player I is to win the game it must end at an inaccessible cardinal $\beta<\kappa$ such that $\sup _{i<\beta} p_{i}=\beta$. Let $p=\bigcup_{i<\beta} p_{i}$. The set $C=\bigcup_{i<\beta} C_{i}$ is a closed unbounded subset of $\beta$. If $p \cap C \neq \varnothing$, then there must exist an ordinal $i<\beta$ such that $p_{i} \cap C_{i} \neq \varnothing$. 
But this would mean that $q_{i} \Vdash$ "U $\dot{G} \cap \dot{C} \neq \varnothing$ ", which is a contradiction. Thus $p$ must be nonstationary and therefore $p$ is a condition in $T_{1}(E)$. Since $p$ extends the previous moves we have described a winning strategy for player II.

Somewhat surprisingly, the key to our main result is the following lemma, which is merely a game version of the fact that $T_{1}(E)$ has $\kappa$-branches. But we need to define a revised version of the game, since comparing with the proof of Lemma 2 , playing first at limits seems to make a difference. Let the game $\mathcal{G}_{\alpha}^{*}(P)$ be exactly as $\mathcal{G}_{\alpha}(P)$ except that player II plays first at limits whereas player I still makes the first move of the game.

Lemma 3. Player II has a winning strategy in $\mathcal{G}_{\kappa}^{*}\left(T_{1}(E)\right)$. Furthermore if $S \subseteq E$ is unbounded, then the winning strategy is definable without reference to $E$ but only to $S$.

Proof. At successor stages the strategy can be arbitrary, except that we make sure that the sequence played is strictly increasing. Suppose that player II is to make her move $q_{i}$ where $i<\kappa$ is a limit ordinal. Let $q=\bigcup_{j<i} q_{j}$ and define $\gamma_{i}=\sup q$. The strategy is simply to let $q_{i}=q \cup \min \left(E \backslash\left(\gamma_{i}+1\right)\right)$, always avoiding "closure" at limits. We need to check that $q_{i}$ really is a condition in $T_{1}(E)$ assuming that the strategy has been followed so far. If $i$ is not inaccessible there is nothing to check. But if $i$ is inaccessible, then $\mathrm{cf} i>\omega$ and thus $\left\{\gamma_{j}: j<i\right.$ is a limit ordinal $\}$ is a club in the complement of $q$.

For the second part involving $S$ we just need to note that we can equally well use choices such as $q_{i}=q \cup \min \left(S \backslash\left(\gamma_{i}+1\right)\right)$ and give a definition for a winning strategy using $S$ as the only parameter.

We will need the second part of the previous lemma as a formal way of saying that the winning strategy is simple enough to be preserved in iterations.

\section{THE BASIC ITERATION}

Let $\kappa$ be a measurable cardinal. We shall define an iteration of forcing notions of the form $T_{1}(E)$. Let $P=P_{\kappa^{+}}$be an iteration of the sequence $\left(\dot{Q}_{\alpha}: \alpha<\kappa^{+}\right)$, which we shall define inductively simultaneously with a sequence $\left(\tau_{\alpha}: \alpha<\kappa^{+}\right)$. Formally $P$ is an iteration of length $\kappa^{+}$but $P$ can be thought of as an Easton support iteration of length $\kappa+1$. We have chosen to "fold out" the "small" iterations that would form the single steps in an iteration of length $\kappa+1$.

Note also that we use a slightly more general interpretation of the notion of an iteration (iterated forcing) as is customary. A condition $p \in P$ is a partial function on $\kappa^{+}$, and we identify the support of $p$ with the domain of $p$. As usual we require that $p(\alpha)$ is a $P_{\alpha}$-name and that $p \mid \alpha \Vdash$ " $p(\alpha) \in \dot{Q}_{\alpha}$ ". The (only) nonstandard feature is that we explicitly specify which $P_{\alpha}$-names can appear as $p(\alpha)$ rather than considering all names up to equivalence. Of course care has to be taken since many basic properties of iterations rely on a rich enough variety of names appearing as $p(\alpha)$. We shall discuss this in greater detail after listing some fundamental properties of $P$ below.

For limit ordinals $\alpha \leq \kappa^{+}$we let $P_{\alpha}$ be a direct limit when $\alpha$ is regular or when cf $\alpha$ is Mahlo and $\operatorname{cf} \alpha \leq \alpha \leq(\operatorname{cf} \alpha)^{+}$. All other limits are inverse. So for Mahlo cardinals $\beta \leq \kappa$ the coordinates $\beta$ through $\beta^{+}$represent a "single step" consisting of an iteration of length $\beta^{+}$with supports of cardinality less than $\beta$. 
For a given ordinal $\alpha$, whenever there is a Mahlo cardinal $\beta \leq \kappa$ such that $\beta \leq \alpha<\beta^{+}$we shall require at each step of the inductive definition that:

(i) $\quad \tau_{\alpha}$ is a $P_{\alpha}$-name for a subset of $\beta$ containing all singular ordinals.

(ii) $\dot{Q}_{\alpha}$ is a $P_{\alpha}$-name for $T_{1}\left(\tau_{\alpha}\right)$ or for the trivial forcing notion $\{\varnothing\}$.

(iii) $p(\alpha)$ is a $P_{\beta}$-name for every $p \in P_{\alpha+1}$.

(iv) For every $p \in P_{\alpha}$ and $P_{\alpha}$-name $\pi$ such that $p \Vdash_{\alpha}$ " $\pi \in \dot{Q}_{\alpha}$ " there is exactly one $q \in P_{\alpha+1}$ such that $q \mid \alpha=p$ and $\Vdash_{\alpha}$ " $q(\alpha)=\pi "$.

Furthermore we require that $p(\alpha)$ is undefined for every $p \in P_{\alpha+1}$ if $\alpha \notin\left[\beta, \beta^{+}\right.$) for any Mahlo cardinal $\beta \leq \kappa$. Thus we can also consider $\tau_{\alpha}$ and $\dot{Q}_{\alpha}$ to be undefined or trivial for such ordinals $\alpha$.

In condition (i) the set of all singular ordinals could be replaced by any set which provably is in the weakly compact ideal and whose definition is absolute enough. It would be tempting to require only that $\tau_{\alpha}$ is a name for an unbounded subset of $\beta$, but due to the restrictions imposed by condition (iii) technical difficulties arise in showing the iterability of strategical closure (e.g. Lemma 5).

In the formulation of condition (ii) the occurrence of the trivial forcing notion is also a technicality. Allowing $\dot{Q}_{\alpha}$ to be a name for $\{\varnothing\}$ ensures that there exist iterations $P$ satisfying conditions (i) (iv). Note also that the method for choosing the $\tau_{\alpha}$ does not affect the existence. Condition (iv) guarantees that $P_{\alpha+1}$ actually is an iteration in the sense that $\left(\dot{Q}_{\alpha}\right)_{G}=\left\{p(\alpha)_{G}: p \in P_{\alpha+1}, p \mid \alpha \in G\right\}$ for every $P_{\alpha}$-generic set $G$. But it is not immediately clear that both (iii) and (iv) may be satisfied unless $\dot{Q}_{\alpha}$ is trivial. However, we shall see later that trivial $\dot{Q}_{\alpha}$ do not need to occur at relevant parts of the iteration.

Notice that condition (iii) makes sense since $P_{\beta} \subseteq P_{\alpha}$ by our way of representing iterations and we assume that names are constructed in such a way that $P_{\beta} \subseteq P_{\alpha}$ implies that every $P_{\beta}$-name also is a $P_{\alpha}$-name. Conditions (iii) and (iv) make the part between $\beta$ and $\beta^{+}$resemble a product rather than an iteration in the strict sense, as we shall see later in the proof of Lemma 4. If (iii) would refer to $P_{\alpha}$-names instead of $P_{\beta}$-names, then our iteration would conform to the strict definition of an iteration commonly presented in the literature.

Before specifying $P$ in greater detail, we shall discuss some properties of any $P$ satisfying the above requirements. Strictly speaking, condition (ii) does not make sense unless we know that the iteration up to $\alpha$ preserves the inaccessibility of $\beta$, as is the case of course. We shall assume that for fixed $\left(\dot{Q}_{\alpha}: \alpha<\kappa^{+}\right)$any $P_{\alpha}$ has minimal rank and the GCH holds in the ground model.

Then the first observations are that $P_{\alpha} \subseteq V_{\alpha}$ for any inaccessible cardinal $\alpha \leq \kappa$ and that for Mahlo cardinals $\beta$ the iteration $P_{\beta}$ satisfies the $\beta$-cc and thus $\left|P_{\beta+}\right| \leq$ $\beta^{+}$. In the standard arguments needed to verify these properties of $P$ the supports play a central role.

We say that a set $A \subseteq \kappa^{+}$is complete if $\kappa \subseteq A$ and $p \mid A \in P$ for every condition $p \in P$. For a complete set $A$ we write $P_{A}=\{p \mid A: p \in P\}=\{p \in P: \operatorname{dom} p \subseteq A\}$ in conformance standard with notation such as $P_{\alpha}$. For complete sets $A$ and $B$ such that $A \subseteq B, P_{A}$ is a complete suborder of $P_{B}$. This is fairly easy to check using the fact that condition (iii) in the case $\beta=\kappa$ guarantees that $p \mid A \leq p$ for any $p \in P_{B}$.

We shall later construct a complete set by induction. To illustrate the simple idea behind this construction, suppose that $\kappa \subseteq A \subseteq \alpha<\kappa^{+}$and that $A$ is complete. The set $A \cup\{\alpha\}$ may fail to be complete because there exists a condition $p \in P$ 
such that $p \mid A \Downarrow$ " $p(\alpha) \in \dot{Q}_{\alpha}$ " even though $p \in P$ implies that $p \mid \alpha \Vdash$ " $p(\alpha) \in \dot{Q}_{\alpha}$ ". But if $\tau_{\alpha}$ happens to be a $P_{A}$-name, which may occur since $P_{A} \subseteq P_{\alpha}$, then also $A \cup\{\alpha\}$ is complete.

Let $A \subseteq \kappa^{+}$be either a complete set or any ordinal below $\kappa^{+}$. (Of course ordinals in $\left[\kappa, \kappa^{+}\right)$are also complete sets.) For the quotient or "tail" we fix the following explicit presentation: $P^{A}=\{p \backslash(p \mid A): p \in P, p \mid A \in G\}$, where $G$ is generic for $P_{A}$. Furthermore if $\alpha \leq \kappa^{+}$we let $P_{\alpha}^{A}=\left\{p \backslash(p \mid A): p \in P_{\alpha}, p \mid A \in G\right\}$ and thus $P_{\alpha}^{A}=P_{\alpha}^{A \cap \alpha}$. For conditions in $P^{A}$ we have $f \leq g$ if and only if there is $p \in G$ such that $p \cup f \leq p \cup g$, both being conditions in $P$.

Let $\dot{P}_{\alpha}^{A}$ denote the $P_{A \cap \alpha}$-name for $P_{\alpha}^{A}$ that resembles $P$. For example, if names are viewed as sets of pairs, then we have $\dot{P}_{\alpha}^{A}=\left\{\left(p \mid A, p_{\alpha}^{A}\right): p \in P_{\alpha}\right\}$, where $p_{\alpha}^{A}$ is a canonical $P_{A \cap \alpha}$-name for $p \backslash(p \mid A)$. With this definition, $P_{\alpha}$ is isomorphic to the two-step iteration $P_{A \cap \alpha} * \dot{P}_{\alpha}^{A}$.

Lemma 4. For any Mahlo cardinal $\beta \leq \kappa$ the iteration $P_{\beta^{+}}$satisfies the $\beta^{+}$-cc.

Proof. Since $P_{\beta}$ satisfies even the $\beta$-cc, it suffices to show that $P_{\beta^{+}}^{\beta}$ satisfies the $\beta^{+}$-cc in $V^{P_{\beta}}=V\left[G_{\beta}\right]$. Consider $f \in P_{\beta^{+}}^{\beta}$ and let $s(\alpha)=\sup \left\{\xi+1: \xi \in f(\alpha)_{G_{\beta}}\right\}$ for $\alpha \in \operatorname{dom} f$ (for $f(\alpha)_{G_{\beta}}=\varnothing$, let $s(\alpha)=0$ ). Note that $f(\alpha)$ is a $P_{\beta}$-name by condition (iii). Let $i(f)$ be the characteristic function of the set $\bigcup_{\beta \leq \alpha<\beta^{+}}(\{\alpha\} \times$ $\left.f(\alpha)_{G_{\beta}}\right)$ seen as a subset of $\bigcup_{\beta \leq \alpha<\beta^{+}}(\{\alpha\} \times s(\alpha))$.

Since $P_{\alpha}$ is a direct limit for any $\alpha \in\left(\beta, \beta^{+}\right)$such that $\operatorname{cf} \alpha=\beta$, it is obvious that $i(f)$ has cardinality less than $\beta$. If $f$ and $g$ are compatible, then there exists a partial function $s:\left(\beta^{+} \backslash \beta\right) \times \beta \rightarrow 2$ of cardinality less than $\beta$ such that $i(f) \cup i(g) \subseteq s$ and thus standard arguments (using $i$ as a complete embedding) can be used to verify the $\beta^{+}$-cc.

It may seem somewhat superfluous to formulate the next lemma using games, since we are after all dealing with rather simple closure properties (see the remark before Lemma 11). But we gain in exactness what we lose in transparency of the intuitive ideas. We shall also later be proving a similar but slightly more involved result where Lemma 3 really is needed, so it will be convenient to be able to refer to the proof presented below. Let $\beta \leq \kappa$ be a Mahlo cardinal and suppose that there is a winning strategy $s:\left[P^{\beta^{+}}\right]^{<\beta^{+}} \rightarrow P^{\beta^{+}}$for player II in $\mathcal{G}_{\beta^{+}}^{*}\left(P^{\beta^{+}}\right)$. We say that $s$ is uniform if

(v) $\operatorname{dom}\left(s\left(p_{i}: i<\xi\right)\right)=\bigcup_{i<\xi} \operatorname{dom} p_{i}$,

(vi) $\left(s\left(p_{i}: i<\xi\right)\right) \mid \gamma=s\left(p_{i} \mid \gamma: i<\xi\right)$ for all $\gamma<\kappa^{+}$

for every $\xi<\beta^{+}$and sequence $\left(p_{i}: i<\xi\right)$ in $P^{\beta^{+}}$.

Lemma 5. For every Mahlo cardinal $\beta \leq \kappa$ there exists a uniform winning strategy $s:\left[P^{\beta^{+}}\right]^{<\beta^{+}} \rightarrow P^{\beta^{+}}$for player II in $\mathcal{G}_{\beta^{+}}^{*}\left(P^{\beta^{+}}\right)$.

Proof. We shall construct $s \mid\left[P_{\alpha}^{\beta^{+}}\right]^{<\beta^{+}}$by induction on $\alpha \in\left[\beta^{+}, \kappa^{+}\right]$. In addition we note that the construction in fact makes $s$ a mapping into $P$, i.e. into $\{p \in P$ : $\left.\operatorname{dom} p \subseteq\left[\beta^{+}, \kappa^{+}\right)\right\} \subseteq P^{\beta^{+}}$. The case $\alpha=\beta^{+}$is trivial.

Suppose that $\alpha=\gamma+1$. We assume that there exists a Mahlo cardinal $\mu$ such that $\mu \leq \alpha<\mu^{+}$since otherwise construction is again trivial. Given a sequence $\left(p_{i}: i<\xi\right)$ for which we are required to find a value for $s$ to assign, we use a 
winning strategy in $G_{\beta^{+}}^{*}\left(T_{1}\left(\tau_{\gamma}\right)\right)$ given by Lemma 3 (even for a longer game) or alternatively the fact that $\dot{Q}_{\gamma}$ is a name for $\{\varnothing\}$.

We may assume that $p_{i}(\gamma)$ is defined for every $i<\xi$. The sequence $\left(p_{i}(\gamma): i<\xi\right)$ is an element of $V^{P_{\beta}+}$ and each $p_{i}(\gamma)$ is a $P_{\mu}$-name. Since $\tau_{\gamma}$ is a $P_{\gamma^{-n a m e ~ w e ~ c o u l d ~}}$ not expect to find a suitable $P_{\mu}$-name using the mere existence of a strategy; note that condition (iii) requires $s\left(\left(p_{i}: i<\xi\right)\right)(\gamma)$ to be a $P_{\mu}$-name. But by condition(i) and the fact that $V^{P_{\mu}}$ and $V^{P_{\gamma}}$ agree about all cofinalities, we can apply Lemma 3 with $S$ the set of singular ordinals. Thus we can find a $P_{\mu}$-name $\rho$ for the value that the strategy (or simple definition, looking at the proof of Lemma 3) gives for $\left(p_{i}(\gamma): i<\xi\right)$. By condition (iv) the correct choice of $\rho$ allows us to put $s\left(\left(p_{i}: i<\xi\right)\right)=s\left(\left(p_{i} \mid \gamma: i<\xi\right)\right) \cup(\gamma, \rho)$.

Suppose now that $\alpha$ is a limit ordinal and that $P_{\alpha}$ is a direct limit. Then also $P_{\alpha}^{\beta^{+}}$is a direct limit and $\mathrm{cf} \alpha>\beta^{+}$, allowing us to put

$$
s\left|\left[P_{\alpha}^{\beta^{+}}\right]^{<\beta^{+}}=\bigcup_{\beta^{+} \leq \gamma<\alpha} s\right|\left[P_{\alpha}^{\beta^{+}}\right]^{<\beta^{+}},
$$

which is also the only possible choice due to the uniformity requirement. If $P_{\alpha}$ is not a direct lim2it, then we also have to deal with sequences $\left(p_{i}: i<\xi\right)$ for which $\bigcup_{i<\xi} \operatorname{dom} p_{i}$ is unbounded in $\alpha$. We know that $P_{\alpha}$ is an inverse limit, but we need to check that also $P_{\alpha}^{\beta^{+}}$is inverse. To be more exact we must find a condition $p \in P_{\alpha}^{\beta^{+}}$such that

$$
p\left|\gamma \leq s\left(p_{i} \mid \gamma: i<\xi\right) \leq p\right| \gamma \text { for all } \gamma \in\left[\beta^{+}, \alpha\right)
$$

to be able to put $s\left(p_{i}: i<\xi\right)=p$. But this is possible by standard arguments (see [1, Th. 5.5] or [4) since $P_{\beta^{+}}$satisfies the $\beta^{+}$-cc, $P_{\gamma}$ is an inverse limit for every limit ordinal $\gamma \in\left(\beta^{+}, \alpha\right)$ such that $\mathrm{cf} \gamma<\beta^{+}$, and condition (iv) again guarantees every appropriate $P_{\beta^{+}}$-name to be represented at coordinates $\alpha \in\left[\beta^{+}, \alpha\right)$.

The previous lemma immediately yields the following property of $P$ : If $\beta \leq \kappa$ is a Mahlo cardinal, then $V^{P}$ and $V^{P_{\beta}+}$ have the same subsets of $\beta$. Thus $V^{P}$ and $V^{P_{\kappa}}$ have the same bounded subsets of $\kappa$.

\section{Details OF THE BASIC ITERATION}

We shall now continue with the definition of the iteration. Recall that GCH holds in the ground model. For each Mahlo cardinal $\beta$ below $\kappa$ we fix $\left(\tau_{\alpha}: \beta \leq\right.$ $\alpha<\beta^{+}$) in such a way that an equivalent representative of every name conforming to condition (i) of Section 4 occurs cofinally often. This is possible by the basic properties of $P$ that we have checked so far. We shall also assume that $\dot{Q}_{\alpha}$ is a name for $T_{1}\left(\tau_{\alpha}\right)$ whenever possible and that $\dot{Q}_{\alpha}$ and the names that will constitute the set $\left\{p(\alpha): p \in P_{\alpha+1}\right\}$ are chosen using some fixed definition.

Let $j: V \rightarrow M$ be given by a normal measure on $\kappa$. We then let $\left(\tau_{\alpha}: \kappa \leq\right.$ $\alpha<\kappa^{+}$) be the sequence in $M$ represented by the function mapping each Mahlo cardinal $\beta<\kappa$ to $\left(\tau_{\alpha}: \beta \leq \alpha<\beta^{+}\right)$. For every $P$-name of a subset of $\kappa$ there is an equivalent representative in $M$, due to $P$ satisfying the $\kappa^{+}$-cc. Thus it is also true in $V$ that $\left(\tau_{\alpha}: \kappa \leq \alpha<\kappa^{+}\right)$enumerates all names up to equivalence.

It follows from the way we picked the names $\tau_{\alpha}$ that $j(P)_{\kappa^{+}}=P$. Arguing in $M$ we know that $j(P)$ satisfies the $j\left(\kappa^{+}\right)$-cc and $|P|=\kappa^{+}$, so up to equivalence there are only $j\left(\kappa^{+}\right)$many $P$-names for antichains of $j(P)^{\kappa^{+}}$in $M$. But $\left|j\left(\kappa^{+}\right)\right|=\kappa^{+}$ 
(even though $j(\kappa)>\kappa^{+}$), so in $V$ we can fix an enumeration $\left(\dot{A}_{\alpha}: \kappa \leq \alpha<\kappa^{+}\right)$of these names. We shall also assume that every name appears cofinally often in the enumeration.

To make subsequent definitions easier to formulate and grasp, we shall make some further assumptions about the sequences $\left(\tau_{\alpha}: \beta \leq \alpha<\beta^{+}\right)$of names. We let them enumerate all names (of subsets of $\beta$ ) with a simple enough structure. Such names are often called nice names. Then given any complete set $A \subseteq \eta<\kappa^{+}$and any $P_{A^{-}}$-name $\tau$ for a subset of $\kappa$ there will be an ordinal $\alpha \in\left[\eta, \kappa^{+}\right)$such that $\tau_{\alpha}$ is equivalent to $\tau$, and $\tau_{\alpha}$ actually is a $P_{A}$-name.

Using Lemma 5 we fix a $P$-name in $M$ for a uniform winning strategy for player II in $\mathcal{G}_{\kappa^{+}}^{*}\left(j(P)^{\kappa^{+}}\right)$. We shall inductively define a set $A$ (of coordinates) such that $\kappa \subseteq A \subseteq \kappa^{+}$simultaneously with a sequence $\left(\dot{f}_{\alpha}: \kappa \leq \alpha<\kappa^{+}\right)$of $P$-names for conditions in $j(P)^{\kappa^{+}}$. The individual choices in the construction take place in $M$, but the construction itself must be carried out in $V$ as it will refer to the sequence $\left(\dot{A}_{\alpha}: \kappa \leq \alpha<\kappa^{+}\right)$.

The induction is on $\alpha$ where $\kappa \leq \alpha<\kappa^{+}$. We shall try to make sure that at each step in the construction

(i) $A \cap \alpha$ is a complete set of coordinates

and the following holds in any generic extension of $M$ by $P$ (dropping the dot from a name indicates interpretation):

(ii) $\left(f_{i}: \kappa \leq i<\alpha\right)$ is a decreasing sequence forming the moves of player I in an instance of $\mathcal{G}_{\kappa^{+}}\left(j(P)^{\kappa^{+}}\right)$where II uses the chosen strategy;

(iii) $f_{\alpha} \in j\left(P_{A \cap(\alpha+1)}\right)^{\kappa^{+}}$;

(iv) if $A_{\alpha}$ is a maximal antichain of $j\left(P_{A \cap \alpha}\right)^{\kappa^{+}}$, then $f_{\alpha} \leq g$ for some $g \in A_{\alpha}$;

(v) if $\tau_{\alpha}$ is a $P_{A \cap \alpha}$-name, then $f_{\alpha}$ decides " $\kappa \in j\left(\tau_{\alpha}\right)$ ";

(vi) if $\alpha \in A$, then $j(\alpha) \in \operatorname{dom} f_{\alpha}$ and $f_{\alpha}(j(\alpha))$ is a $P_{A \cap(\alpha+1)}$-name such that

$$
\Vdash " f_{\alpha}(j(\alpha))=\bigcup\left\{u(\alpha): u \in \dot{G}_{A \cap(\alpha+1)}\right\} \cup\{\kappa\} " .
$$

Finally we shall require that

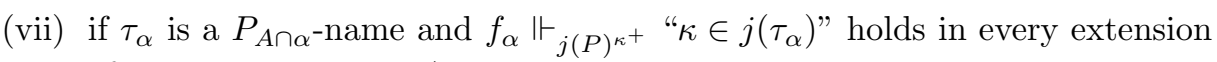
of $M$ by $P$, then $\alpha \in A$.

Condition $(\mathrm{v})$ is stated in an informal way. Since $j\left(\tau_{\alpha}\right)$ is a $j\left(P_{A \cap \alpha}\right)$-name the statement " $\kappa \in j\left(\tau_{\alpha}\right)$ " is in the forcing language of $j\left(P_{A \cap \alpha}\right)$. But looking at (iii)] realises that $j\left(\tau_{\alpha}\right)$ has to be replaced (arguing in $\left.M\right)$ by a $P$-name for a $j\left(P_{A \cap \alpha}\right)^{\kappa^{+}}$name which is forcing equivalent to the original $j\left(\tau_{\alpha}\right)$. The interpretation in $M^{P}$ of this name then appears in the formal forcing statement of (v) which also appears in condition (vii)

In condition (vi)] $\dot{G}_{A \cap(\alpha+1)}$ denotes the canonical name for a $P_{A \cap(\alpha+1)}$-generic set and thus each $s(\alpha)$ is a $P_{\kappa}$-name. Thus the forcing relation referred to is obviously that of $P_{A \cap(\alpha+1)}$ or any forcing notion that includes $P_{A \cap(\alpha+1)}$ as a complete suborder. We must let $f_{\alpha}(j(\alpha))$ be a $j(P)_{j(\kappa)}$-name if we are to also satisfy (iii) But this is no problem since $P_{A \cap(\alpha+1)}$ is a complete suborder of $P$ and $P=j(\bar{P})_{\kappa}$.

The conditions above are stated in a form that keeps the following kind of argument in mind. To pick $\dot{f}_{\alpha}$, fix some $G$ which is $P$-generic over $M$. Working in $M[G]$ it is possible to pick a function $f_{\alpha} \in M[G]$ that satisfies conditions (ii) (vi) 
Since $G$ is arbitrary, there has to be a $P$-name $\dot{f}_{\alpha}$ for such an object $f_{\alpha}$. Finally one notices that it is possible to keep (vii) true without violating (i) or (vi).

Once the formal interpretation of the above conditions is made clear, it should not be hard to verify that they indeed can be kept true during an inductive construction of $A$. For (iii) we make use of the fact that the strategy is uniform. To meet (vii) we can still drop $\alpha$ from $A$ whenever $\tau_{\alpha}$ is not a $P_{A \cap \alpha}$-name and this automatically takes care of condition (i) and makes meeting condition (vi) possible. To see that both (iii) and (vi) can be kept true in the case $\alpha \in A$ we need to note e.g. that being 1-closed is an upwards absolute notion.

One might notice that if $\left(\tau_{\alpha}: \kappa \leq \alpha<\kappa^{+}\right)$is not carefully defined and the inductive statement is interpreted literally, the set $A$ constructed might very well end up being $\kappa$. However, fixing a specific name construction and making sure that every "nice name" is listed in $\left(\tau_{\alpha}: \kappa \leq \alpha<\kappa^{+}\right)$cofinally often (as we assume is the case), it is fairly easy to see that $A$ must be an unbounded subset of $\kappa^{+}$. The careful listing of names is necessary for the easy but crucial argument of Lemma 13 at the end of the paper.

This completes the definition of our basic forcing notion; it will be $P_{A}$. Let $\dot{H}$ be a $P$-name for the set $\left\{g \in j\left(P_{A}\right)^{\kappa^{+}}: f_{\alpha} \leq g\right.$ for some $\left.\alpha<\kappa^{+}\right\}$. In the lemma below we identify $G * H$ with the corresponding subset of $j\left(P_{A}\right)$.

Lemma 6. For every set $G \subseteq P$ which is generic for $P$ over $V$, the set $H=\dot{H}_{G}$ is generic for $j\left(P_{A}\right)^{\kappa^{+}}$over $M[G]$. Furthermore $j: V \rightarrow M$ can be extended to an elementary embedding $j: V\left[G_{A}\right] \rightarrow M[G][H]$ such that $j\left(G_{A}\right)=G * H$.

Proof. Since $j\left(P_{A}\right)^{\kappa^{+}}$is $j\left(\kappa^{+}\right)$-cc, every maximal antichain of $j\left(P_{A}\right)^{\kappa^{+}}$is already a subset of $j(P)_{j(A) \cap \eta}^{\kappa^{+}}$for some $\eta<j\left(\kappa^{+}\right)$. Since $j\left[\kappa^{+}\right]$is unbounded below $j\left(\kappa^{+}\right)$, we have $j(P)_{j(A) \cap \eta}^{\kappa^{+}} \subseteq j\left(P_{A \cap \alpha}^{\kappa^{+}}\right)$for some $\alpha<\kappa^{+}$. Thus condition (iv) ensures that $H$ will intersect every dense set in $M[G]$, as $\left(\dot{A}_{\alpha}: \kappa \leq \alpha<\kappa^{+}\right)$lists every name cofinally often.

Let $p \in G_{A}$. To show that $j: V \rightarrow M$ can be extended we are required to show that $j(p) \mid \kappa^{+} \in G$ and $j(p) \backslash\left(j(p) \mid \kappa^{+}\right) \in H$. But $j(p)\left|\kappa^{+}=p\right| \kappa$, so the first part is clear. For the second part we note that $|\operatorname{dom} p|<\kappa$ whereby $j(\operatorname{dom} p) \subseteq j[A]$. Since $j(p)(j(\alpha))=p(\alpha)$ for every $\alpha \in \operatorname{dom} p$ condition (vi) gives $j(p) \backslash\left(j(p) \mid \kappa^{+}\right) \in H$.

In the extension $V^{P_{A}}$ we can use the forcing relation of $P^{A}$ to define a filter $F$ over $\kappa$ by putting

$$
X \in F \text { if and only if } \Vdash_{\kappa^{+} \backslash A} \text { " } \kappa \in j(\check{X}) \text { ". }
$$

We will see that $F$ is the weakly compact filter of $V^{P_{A}}$. Note that the extended embedding $j$ above should be thought of as defined through the $P$-name $\dot{H}$ as in Lemma 6.

\section{Preservation of Weak Compactness}

We shall now show that the iteration $P_{A}$ defined in the previous section preserves the weak compactness of the measurable cardinal $\kappa$.

Lemma 7. For every ordinal $\eta \in\left[\kappa, \kappa^{+}\right)$player II has a winning strategy in $\mathcal{G}_{\kappa}^{*}\left(P^{A \cap \eta}\right)$. 
Proof. We can construct a uniform winning strategy $s$, defining $s \mid\left[P_{\alpha}^{A \cap \eta \cap \alpha}\right]^{<\kappa}$ by induction on $\alpha \in\left[\kappa, \kappa^{+}\right]$in a similar manner as in the proof of Lemma 5 . The construction takes place in $V^{P_{A \cap \eta}}$ and relies heavily on the fact that $V^{P_{A \cap \eta}}$ has the same bounded subsets of $\kappa$ as $V^{P_{\kappa}}$.

When $\alpha \geq \eta$ the winning strategies, the games played, and the whole construction are all in $V^{P_{A \cap \eta}}$, and the situation is as simple as it is in the proof of Lemma 5 But for $\alpha \in[\kappa, \eta)$ we are actually talking about games played in $V^{P_{A \cap \alpha}}$. But since the bounded subsets of $\kappa$ are the same, a winning strategy from $V^{P_{A \cap \alpha}}$ is still a winning strategy in $V^{P_{A \cap \eta}}$.

The initial step $\alpha=\kappa$ is trivial. Also the successor step $\alpha=\gamma+1$ is trivial whenever $\gamma \in A \cap \eta$. The nontrivial successor steps work since we are dealing with decreasing sequences $\left(p_{i}: i<\xi\right)$ of length less than $\kappa$. Such a sequence is an element of $V^{P_{\kappa}}$, and thus we can apply Lemma 3 with $S$ the set of singular limits.

The limit step in which $P_{\alpha}^{A \cap \eta \cap \alpha}$ is a direct limit also covers the trivial case where $\alpha \backslash(A \cap \eta)$ is a bounded subset of $\alpha$. If $\alpha \backslash(A \cap \eta)$ is unbounded, then also $P_{\alpha}$ must be a direct limit, in which case cf $\alpha=\kappa$ or $\alpha=\kappa^{+}$. Then the strategy at stage $\alpha$ is simply the union of the strategies of the previous stages.

Finally suppose $\alpha$ is a limit ordinal and $P_{\alpha}^{A \cap \eta \cap \alpha}$ is not a direct limit. Potentially a problem arises here since $P_{A \cap \eta}$ most likely does not satisfy the $\kappa$-cc. But $\mathrm{cf} \alpha<\kappa$ and thus any sequence $\left(s\left(p_{i} \mid \gamma: i<\xi\right): \gamma<\alpha\right)$ that we need to consider is already in $V^{P_{\kappa}}$. Since $s\left(p_{i} \mid \gamma: i<\xi\right) \in P_{\gamma}^{A \cap \eta \cap \gamma}$ for each $\gamma$, we can find a $P_{\kappa}$-name representing the entire sequence and the standard argument mentioned in the proof of Lemma 5 goes through.

The previous lemma enables us to use the following form of a result known from the literature. Note that $V_{\kappa}$ is absolute with respect to the models under consideration.

Lemma 8. Let $\kappa$ be an inaccessible cardinal, let $\phi$ be $a \Pi_{1}^{1}$-sentence, and let $U \subseteq V_{\kappa}$ be such that $\left\langle V_{\kappa}, \in, U\right\rangle \models \phi$. Consider a forcing notion $P$ such that player II has a winning strategy in $\mathcal{G}_{\kappa}^{*}(P)$. Then $\left\langle V_{\kappa}, \in, U\right\rangle \models \phi$ holds in $V^{P}$.

Lemma 9. In the generic extension $V^{P_{A}}$ every set $E \in F$ is weakly compact.

Proof. Let $\phi$ be a $\Pi_{1}^{1}$-sentence, let $\dot{U}$ be a $P_{A}$-name for a subset of $V_{\kappa}$ of the generic extension, and suppose that

$$
p \Vdash "\left\langle V_{\kappa}, \in, \dot{U}\right\rangle \models \phi "
$$

for some $p \in P_{A}$. Let $\dot{E}$ be a $P_{A}$-name for an element of $F$. The lemma will be proved if we can find a condition $q \leq p$ such that

$$
q \Vdash_{P_{A}} \text { " } \alpha \in \dot{E} \text { and }\left\langle V_{\alpha}, \in, \dot{U} \cap V_{\alpha}\right\rangle \models \phi "
$$

for some ordinal $\alpha<\kappa$.

Pick a generic set $G$ for $P$ over $V$ and $M$ such that $p \in G_{A}$. Notice that $V_{\kappa}$ is absolute between all the models under discussion that extend $M\left[G_{\kappa}\right]$. We can fix an ordinal $\eta<\kappa^{+}$such that $\dot{U}$ is a $P_{A \cap \eta^{-n a m e . ~ B e c a u s e ~} A \cap \eta \in M \text { the }}$ model $M\left[G_{A \cap \eta}\right]$ is a properly defined generic extension. In $M\left[G_{A \cap \eta}\right]$ it follows that $\left\langle V_{\kappa}, \in, \dot{U}_{G_{A \cap \eta}}\right\rangle \models \phi$ by downwards absoluteness, since the same statement holds in $V\left[G_{A}\right]$ by the fact that $p \in G_{A}$. By Lemma 8 and Lemma 7 the statement holds in $M[G]$ too. Finally, since $j(P)^{\kappa^{+}}$adds no new subsets of $\kappa$ it holds in $M[G][H]$, where $H$ is as in Lemma 6 . 
For our extended elementary embedding $j: V\left[G_{A}\right] \rightarrow M[G][H]$, we have that $j\left(\dot{U}_{G_{A}}\right) \cap V_{\kappa}=\dot{U}_{G_{A}}$ and $\kappa \in j\left(\dot{E}_{G_{A}}\right)$. So by elementarity it is the case in $V\left[G_{A}\right]$ that there exists an ordinal $\alpha<\kappa$ such that

$$
\alpha \in \dot{E}_{G_{A}} \text { and }\left\langle V_{\alpha}, \in, \dot{U}_{G_{A}} \cap V_{\alpha}\right\rangle \models \phi,
$$

and therefore we can find the required condition $q \leq p$ deciding $\alpha$.

For our application of the previous lemma it would have sufficed to prove the case $E=\kappa$, but the proof involving $F$ is illustrative and not significantly more involved.

\section{Consistency of the WeAkly COMPACT IDEAL Being SATURATED}

Since we are going to show that $F$ is the weakly compact filter there is no need to separately check that $F$ indeed is a filter, although it would be fairly straightforward directly from the definition. The following is a special case of a well-known fact.

Lemma 10. Given that $F$ is a filter in $V^{P_{A}}$, then $F$ is saturated.

Proof. Assume that $F$ is a filter. Then a set $X \subseteq \kappa$ is in the dual ideal iff $\Vdash_{\kappa^{+} \backslash A}$ " $\kappa \notin j(\check{X})$ " and $X$ has positive measure iff there exists a condition $f \in P^{A}$ such that $f \vdash_{\kappa^{+} \backslash A}$ " $\kappa \in j(\check{X})$ ". But $P^{A}$ satisfies the $\kappa^{+}$-cc because $P$ does. Hence $F$ must be saturated.

The following two lemmas are intimately connected with the preservation of weak compactness presented in the previous section. If $\kappa$ is weakly compact, then the 1-clubs characterise the ideal $\Pi_{1}^{1}(\kappa)$, but by a result of Sun [7], the 1-clubs may even generate a proper filter without $\kappa$ being weakly compact.

Lemma 11. The dual $\left(\Pi_{1}^{1}(\kappa)\right)^{*} \subseteq F$ in $V^{P_{A}}$.

Proof. By Lemma 9 $k$ is weakly compact in $V^{P_{A}}$, and thus it suffices to show that every 1 -club is in $F$. Let $G$ be generic for $P$ over $V$ and $M$ and let $E$ be a 1-club in $V\left[G_{A}\right]$. Using Lemma 8 (or Lemma 7 directly) together with standard arguments, one sees that $E$ is still stationary in $V[G]$. Thus $E$ is stationary in $M[G]$ and hence in $M[G][H]$. It follows that $\kappa \in j(E)$. Since $G$ can be arbitrary after fixing $G_{A}$ we must have $E \in F$.

Lemma 12. For every $\alpha \in A \backslash \kappa$ the set $E=\bigcup_{s \in G_{A \cap(\alpha+1)}} s(\alpha)_{G_{A \cap \alpha}}$, i.e. the generic branch produced by $T_{1}\left(\left(\tau_{\alpha}\right)_{G_{A \cap \alpha}}\right)$, is a 1-club in $V\left[G_{A}\right]$.

Proof. Obviously $E$ is 1 -closed. We need to show that $E$ is stationary. It suffices to show that $\kappa \in j(E)$, since $\kappa \in j(C)$ for every club $C \in V\left[G_{A}\right]$. By the definition of the extended elementary embedding we have $j(E)=\bigcup_{s \in G * H} s(j(\alpha))_{G * H}$. By condition (vi) in Section 5 we have $\Vdash " \kappa \in f_{\alpha}(j(\alpha))$ ", and $f_{\alpha} \in H$ by definition. Thus $\kappa \in j(E)$.

Lemma 13. $F \subseteq\left(\Pi_{1}^{1}(\kappa)\right)^{*}$ in $V^{P_{A}}$.

Proof. Let $\tau$ be a $P_{A}$-name for a set in $F$. We only need to show that $\tau$ is equivalent to $\tau_{\alpha}$ for some $\alpha \in A \backslash \kappa$ since then $\tau_{G_{A}} \in\left(\Pi_{1}^{1}(\kappa)\right)^{*}$ by Lemma 12. We may assume that $\tau$ is a name for a set containing all singular ordinals below $\kappa$, because the set of singular ordinals is in the weakly compact ideal. 
Since $P_{A}$ satisfies the $\kappa^{+}$-cc there certainly is an ordinal $\alpha \in\left[\kappa, \kappa^{+}\right)$such that $\tau_{\alpha}$ is a $P_{A \cap \alpha}$-name equivalent to $\tau$. Since the interpretation of $\tau$ is in $F$ in every extension by $P_{A}$ we must have $\Vdash_{P} " \kappa \in j\left(\tau_{\alpha}\right)$ ". But then conditions (v) and (vii) together ensure that $\alpha \in A$.

Combining the lemmas presented above with the fact that GCH can be forced to hold while preserving measurability, we obtain Theorem 1 .

The basic forcing notion $P_{A}$ that we defined and the appropriate variations thereof can be used as a building block for longer iterations in exactly the same way as is described in [6]. One then starts with $\kappa$ being measurable of order $\alpha$, where $\alpha<\kappa^{+}$. For instance, if $\kappa$ is measurable of order 2, then every $\beta<\kappa$ that is measurable of order 1 will be weakly compact after an iteration of length $\beta^{+}$that resembles the basic iteration presented in this paper. The entire iteration of length $\kappa^{+}$results in $\kappa$ being weakly compact of order 2 . The details can be extracted from [6]. This establishes yet another analog between the weakly compact hierarchy [5] and the Mahlo hierarchy [2].

\section{REFERENCES}

1. James E. Baumgartner, Iterated forcing, Surveys in Set Theory (A. R. D. Mathias, ed.), London Math. Soc. Lecture Note Ser., vol. 87, Cambridge University Press, 1983, pp. 1-59. MR 823775 (87c:03099)

2. James E. Baumgartner, Alan D. Taylor, and Stanley Wagon, On splitting stationary subsets of large cardinals, J. Symbolic Logic 42 (1977), no. 2, 203-214. MR0505505 (58:21619)

3. Moti Gitik and Saharon Shelah, Less saturated ideals, Proc. Amer. Math. Soc. 125 (1997), 1523-1530. MR.1363421 (97g:03055)

4. Alex Hellsten, Diamonds on large cardinals, Ann. Acad. Sci. Fenn. Math. Diss. 134 (2003). MR:2026390 (2004j:03054)

5. , Orders of indescribable sets, Arch. Math. Logic 45 (2006), no. 6, 705-714. MR 2252250 (2007d:03092)

6. Thomas Jech and W. Hugh Woodin, Saturation of the closed unbounded filter on the set of regular cardinals, Trans. Amer. Math. Soc. 292 (1985), no. 1, 345-356. MR805967(87d:03139)

7. Wenzhi Sun, Stationary cardinals, Arch. Math. Logic 32 (1993), no. 6, 429-442. MR1245524 (94i:03103)

8. Alfred Tarski, Ideale in vollständigen Mengenkörpern. II, Fund. Math. 33 (1945), 51-65. MR0017737 (8:193b)

Finnish Matriculation Examination Board, P.O. Box 50, 00581 Helsinki, Finland

E-mail address: alex.hellsten@iki.fi 